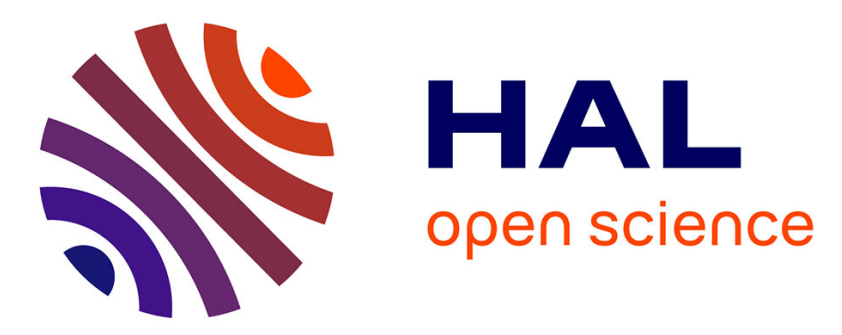

\title{
Evaporative Optical Marangoni Assembly: Tailoring the Three-Dimensional Morphology of Individual Deposits of Nanoparticles from Sessile Drops
}

Manos Anyfantakis, Subramanyan Namboodiri Varanakkottu, Sergii Rudiuk, Mathieu Morel, Damien Baigl

\section{To cite this version:}

Manos Anyfantakis, Subramanyan Namboodiri Varanakkottu, Sergii Rudiuk, Mathieu Morel, Damien Baigl. Evaporative Optical Marangoni Assembly: Tailoring the Three-Dimensional Morphology of Individual Deposits of Nanoparticles from Sessile Drops. ACS Applied Materials \& Interfaces, 2017, 9 (42), pp.37435 - 37445. 10.1021/acsami.7b11547 . hal-01626237

\section{HAL Id: hal-01626237 \\ https://hal.sorbonne-universite.fr/hal-01626237}

Submitted on 30 Oct 2017

HAL is a multi-disciplinary open access archive for the deposit and dissemination of scientific research documents, whether they are published or not. The documents may come from teaching and research institutions in France or abroad, or from public or private research centers.
L'archive ouverte pluridisciplinaire HAL, est destinée au dépôt et à la diffusion de documents scientifiques de niveau recherche, publiés ou non, émanant des établissements d'enseignement et de recherche français ou étrangers, des laboratoires publics ou privés. 
Subscriber access provided by BUPMC - Bibliothèque Universitaire Pierre et Marie Curie

\section{Article}

\section{Evaporative Optical Marangoni Assembly (eOMA): tailoring the three-dimensional morphology of individual deposits of nanoparticles from sessile drops}

Manos Anyfantakis, Subramanyan Namboodiri Varanakkottu, Sergii Rudiuk, Mathieu Morel, and Damien Baigl

ACS Appl. Mater. Interfaces, Just Accepted Manuscript • DOI: 10.1021/acsami.7b11547 • Publication Date (Web): 06 Oct 2017 Downloaded from http://pubs.acs.org on October 9, 2017

\section{Just Accepted}

"Just Accepted" manuscripts have been peer-reviewed and accepted for publication. They are posted online prior to technical editing, formatting for publication and author proofing. The American Chemical Society provides "Just Accepted" as a free service to the research community to expedite the dissemination of scientific material as soon as possible after acceptance. "Just Accepted" manuscripts appear in full in PDF format accompanied by an HTML abstract. "Just Accepted" manuscripts have been fully peer reviewed, but should not be considered the official version of record. They are accessible to all readers and citable by the Digital Object Identifier (DOI $\left.{ }^{8}\right)$. "Just Accepted" is an optional service offered to authors. Therefore, the "Just Accepted" Web site may not include all articles that will be published in the journal. After a manuscript is technically edited and formatted, it will be removed from the "Just Accepted" Web site and published as an ASAP article. Note that technical editing may introduce minor changes to the manuscript text and/or graphics which could affect content, and all legal disclaimers and ethical guidelines that apply to the journal pertain. ACS cannot be held responsible for errors or consequences arising from the use of information contained in these "Just Accepted" manuscripts. 


\title{
Evaporative Optical Marangoni Assembly (eOMA): tailoring the three-dimensional morphology of individual deposits of nanoparticles from sessile drops
}

\author{
Manos Anyfantakis ${ }^{1,2} \uparrow \dagger$, Subramanyan Namboodiri Varanakkottu ${ }^{1,2,3} \uparrow$, Sergii Rudiuk ${ }^{1,2}$, \\ Mathieu Morel ${ }^{1,2}$, and Damien Baigl ${ }^{1,2 *}$ \\ ${ }^{1}$ PASTEUR, Department of chemistry, École normale supérieure, UPMC Univ. Paris 06, \\ CNRS, PSL Research University, 75005 Paris, France \\ ${ }^{2}$ Sorbonne Universités, UPMC Univ. Paris 06, École normale supérieure, CNRS, PASTEUR, \\ 75005 Paris, France \\ ${ }^{3}$ School of Nano Science and Technology, National Institute of Technology Calicut, India
}

\begin{abstract}
We have recently devised the evaporative Optical Marangoni Assembly (eOMA), a novel and versatile interfacial flow-based method for directing the deposition of colloidal nanoparticles (NPs) on solid substrates from evaporating sessile drops, along desired patterns using shaped UV light. Here, we focus on a fixed UV spot irradiation resulting in a cylinder-like deposit of assembled particles and show how the geometrical features of the single deposit can be tailored in three dimensions by simply adjusting the optical conditions or the sample composition, in a quantitative and reproducible manner. Sessile drops containing cationic NPs and a
\end{abstract}


photosensitive surfactant at various concentrations are let evaporated under a single UV beam with a diameter much smaller than that of the drop. After complete evaporation, the geometrical characteristics of the NP deposit are precisely assessed using optical profilometry. We show that both the volume and the radial size of the light-directed NP deposit can be adjusted by varying the diameter or the intensity of the UV beam, or alternatively by changing the concentration of the photosensitive surfactant. Notably, in all these cases, the deposits display an almost constant median height corresponding to a few layers of particles. Moreover, both the radial and the axial extent of the patterns are tuned by changing the NP concentration. These results are explained on the one hand by the correlation between the strength of Marangoni flow, the particle trapping efficiency and the volume of the deposit, and, on the other hand, by the role of evaporation-driven flow in strongly controlling the deposit height. Finally, we extend the versatility of eOMA by demonstrating that NPs down to $30 \mathrm{~nm}$ in diameter can be effectively patterned on glass or polymeric substrates.

Keywords: directed assembly, colloidal patterning, photocontrol, photoisomerization, sessile drop, evaporation, Marangoni flow, Coffee-Ring Effect 


\section{INTRODUCTION}

Colloidal chemistry has currently reached a high level of elaboration, being readily able to deliver nano- and micro-particles with tailored physical and chemical properties such as size, shape and surface chemistry. ${ }^{1}$ At present, perhaps the major effort of material scientists is more and more shifting toward finding ways of organizing colloids in a controlled fashion, a characteristic necessary for applications. ${ }^{2}$ Indeed, colloidal ensembles display much greater structural and functional complexity compared to that of the single particles, ${ }^{3}$ which is demonstrated by the wealth of impressive achievements in the fabrication of functional assemblies and devices, utilized in photonic, ${ }^{4}$ photovoltaic ${ }^{5}$ and sensing applications, ${ }^{6}$ to name a few. The large number of colloid organization strategies reported so far is proportional to the vivid interest shown by scientists. ${ }^{7}$ Whereas elegant patterning methods can lead to assemblies of very detailed characteristics, to achieve a highly complex and well-controlled colloid organization, the trade-off is usually the significant effort needed for the system design and/or the inherent limitations posed by each approach. ${ }^{8}$ Moreover, although powerful methods, such as capillary assembly ${ }^{9}$ or micro-contact printing ${ }^{10}$ have been developed to distribute a large number of colloids (e.g., particles, cells) along predefined patterns with exquisite resolution, ${ }^{11,12}$ controlling the three-dimensional features of a single deposit remains a methodological challenge. As a result, there is a need to develop simple yet robust strategies to tailor the way particles assemble in three dimensions within single deposits.

Exploiting hydrodynamic flows in colloidal dispersions is a prominent example of a potentially simple approach to achieve tailored particle assemblies. For instance, when a volatile colloidal suspension partially wets a solid substrate (i.e., the formed contact angle is less than $90^{\circ}$ ), a gradient in the evaporation rate along the liquid-gas interface is developed, with its maximum being at the pinned contact line. A convective flow toward the contact line 
is thus developed, in order to account for the locally enhanced liquid loss. This flow carries the dispersed particles with it and eventually collects them at the periphery of the wetted area. ${ }^{13}$ This very robust effect has been the basis of a plethora of convective ${ }^{14}$ patterning methods, as it has been exploited in various geometries and numerous liquid-substrate combinations. ${ }^{8}$

Perhaps the simplest case of evaporative particle assembly is a sessile drop of a colloidal dispersion which is drying on a solid substrate. In the common case of a pinned contact line, the evaporation-driven outward flow gathers particles at the drop edge, leading to the characteristic ring-shaped patterns after drying. ${ }^{13,15}$ The most successful strategies for modulating this so-called 'Coffee-Ring Effect' (CRE) are to manipulate the flow patterns inside the drying drop ${ }^{16,17}$ or, alternatively, to exploit the interactions between the particles and the interfaces present in the system. ${ }^{18-22}$ Successful suppression was shown to lead to deposits of various three-dimensional morphologies ranging from thin or thick disks to domes. ${ }^{17,19,23}$

Apart from these relatively simple morphologies mentioned above, more complex patterns have also been observed in evaporating suspension drops. For non-pinned drops, the stick-slip motion of the contact line during drying was shown to lead to a large number of concentric rings in freely evaporating ${ }^{24}$ or geometrically confined ${ }^{25}$ particle-laden drops. In contrast to ring-shaped morphologies, deposition patterns displaying radial asymmetry have also been achieved using drops which were receding during drying, however substrates with patterned wettability had to be used. ${ }^{26}$

The spatial modulation of the hydrodynamic patterns developed within colloidal dispersions is a promising method for organizing particles on solid substrates in a controlled fashion. An early demonstration of tailored colloidal patterning inside a drying aqueous dispersion was reported by Harris et al. ${ }^{27}$ In this very simple strategy, a mask containing a periodic array of holes was placed above the liquid to create an evaporation rate landscape 
consisting of regions of free and hindered evaporation. This led to the formation of colloidal films displaying a periodic height variation, the morphology of which corresponded to the mask design. $^{27,28}$ This approach is remarkable for its robustness, however it has a limited reconfigurability.

Researchers have thus looked for ways to shape deposits from evaporative drops using light, an external and reconfigurable stimulus that can be delivered locally with high spatiotemporal resolution. ${ }^{29}$ A common strategy has consisted in converting light to thermal energy. Indeed, when light irradiation leads to a local temperature rise at the free interface of a liquid, evaporation-driven convective flows and/or thermal Marangoni flows, ${ }^{30}$ arising from surface tension gradients, can emerge. Building on the work of Harris et al. ${ }^{27}$, Georgiadis et al. utilized shaped IR light to locally heat a colloidal suspension film and create variations in the evaporation rate across its surface. The combination of evaporation-driven convective flow and light-induced particle sintering led to the formation of dry films displaying a bespoke topography. ${ }^{31}$ IR-modulated evaporative deposition of organic electronic materials from solutions utilizing a micromirror-based optical setup, has been demonstrated by Salas et al. ${ }^{32}$ Two deposition modes, one based on the evaporation-driven flow and a second one due to thermal and solutal Marangoni stresses were identified. In a recent work, Ta et al. used a high power IR laser beam to locally heat a colloidal suspension drop and drive thermocapillary and evaporation-driven flows that could accumulate particles at selected positions on solid substrates. $^{33}$ A hybrid optoelectric technique was used by Kumar et al. to concentrate microparticles at predefined positions across electrode surfaces after drop evaporation. Application of an AC electric field and IR illumination resulted in the accumulation of the majority of particles at the focal point of the laser beam, due to a multitude of wetting and electrokinetic effects. ${ }^{34}$ Photothermally generated and controlled microbubbles have also been successfully employed to create arbitrary single and multiple particle patterns on plasmonic 
substrates. The microbubbles were able to capture and immobilize the particles on the substrate through the combined action of Marangoni flows, surface tension, gas pressure and adhesion to the substrate. ${ }^{35}$ In a very recent study, Sakaguchi et al. demonstrated the reversible control of particle aggregation in a photosensitive liquid utilizing a combined effect of light-controlled convective flow and viscosity modulation. ${ }^{36}$ However, the majority of these strategies relied on a local increase of temperature, meaning a strong sensitivity to temperature conditions and an applicability restricted to light-absorbing and heat-insensitive samples.

An interesting alternative consists in using photosensitive surfactants, so that lightinduced Marangoni flows can be generated in a quasi-isothermal manner. ${ }^{37-41}$ We recently described a new optofluidic operation where such light-induced surface flows were exploited for depositing particles along complex predefined patterns on a solid surface. ${ }^{42}$ This was achieved in drying drops of colloidal suspensions mixed with a photosensitive surfactant (AzoTAB). ${ }^{37}$ The so-called evaporative Optical Marangoni Assembly (eOMA) ${ }^{42}$ was based on photochemically controlled hydrodynamic flow patterns, in contrast to other particle manipulation approaches relying directly ${ }^{34}$ or indirectly $32,33,35,43$ on photothermal effects or diffusioosmosis-driven flow. ${ }^{44}$ Simultaneous irradiation of the whole drop with diffuse white light and of the drop center with a focused UV beam, resulted in surface tension $(\gamma)$ pattern at the free surface of the drop. The surface tension of the UV-irradiated area $\left(\gamma_{U V}\right)$ was higher than the one corresponding to the areas exposed to white light $\left(\gamma_{V I S}\right)$, owing to the reversible trans cis isomerization of AzoTAB upon UV illumination. ${ }^{41}$ The imposed $\nabla \gamma$ resulted in Marangoni flows at the free surface toward the UV-exposed region. These surface flows, combined with the evaporation-driven radial flow, created a trapping volume within the drying drop, where particles were accumulated until the late stage of evaporation. After complete drying, particles were precisely positioned on the substrate, at and around the UV-irradiated areas. ${ }^{42}$ We have demonstrated that eOMA allowed us to distribute different types of particles along a broad 
variety of desired patterns by using shaped UV irradiation to direct their deposition. The characteristics of individual deposits under each UV-irradiated area were however only partially controlled.

Controlling the three-dimensional features of individual light-driven particle deposits thus appears as a needed methodological improvement for more precisely shaped particle assemblies on a solid surface. Because eOMA was shown as a particularly versatile method for light-directed deposition of particles, here we explored whether it could be exploited for controlling the geometrical characteristics of individual particle deposits produced from evaporating colloidal suspensions under a single UV spot. Our main hypothesis was that the optical (intensity, beam size) as well as the material (photosensitive surfactant and particle concentration, particle and substrate type) parameters should directly influence the process of eOMA through different physicochemical effects. To test this hypothesis, we employed interference microscopy to systematically and precisely quantify the effect of these parameters on the geometrical features of individual light-directed deposits (shape, lateral size, height, volume). Our results showed that the volume and the shape of the deposit can be tuned on demand by the rational selection of experimental conditions, not only improving the performance of eOMA but also bringing a new understanding of the coupling between interfacial flows and particle deposition features in evaporating drops.

\section{RESULTS AND DISCUSSION}

Figure 1a schematically describes our experiment, which is based on a typical eOMA configuration. ${ }^{42}$ An aqueous sessile drop containing cationic NPs and AzoTAB was deposited on a glass coverslip. Although eOMA is compatible with both anionic and cationic particles, we deliberately chose to work with cationic particles to avoid electrostatic adsorption of the 
cationic AzoTAB on the NP surface, which in some specific concentration ranges (much lower than the critical micellar concentration) can have dramatic effects on the deposition process. ${ }^{18,19}$ During drop evaporation, a UV beam was focused at the center of the drop while white light was homogeneously shined on the whole drop. The drop was protected from air currents and it was let dry under controlled temperature and relative humidity conditions. After complete evaporation, a pattern made of NPs and surfactants which were deposited on the glass substrate, was formed. In the majority of our experiments, we fixed the drop volume $(7 \mu \mathrm{L})$, the diameter of NPs $(200 \mathrm{~nm})$, the substrate type and the evaporation conditions (temperature and relative humidity range of $22.0 \pm 0.5{ }^{\circ} \mathrm{C}$ and $45.8 \pm 7.3 \%$, respectively), and we varied the particle concentration $\left(C_{p}\right)$, AzoTAB concentration $\left(C_{s}\right)$, the spot radius (ruv) and the irradiation intensity $\left(I_{U V}\right)$. Optical profilometry was used to precisely extract the three-dimensional characteristics of each individual UV-induced particle deposit as a function of these control parameters.

a

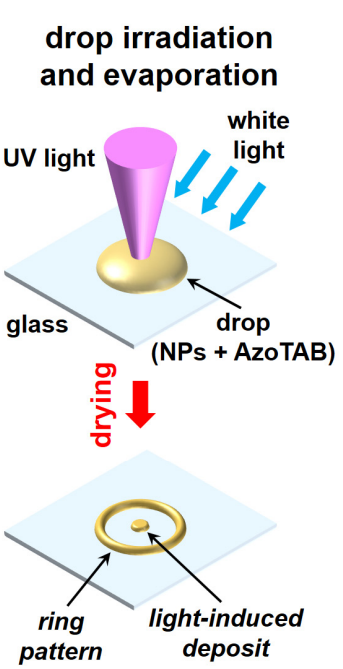

b

imaging of dry pattern

optical profilometry

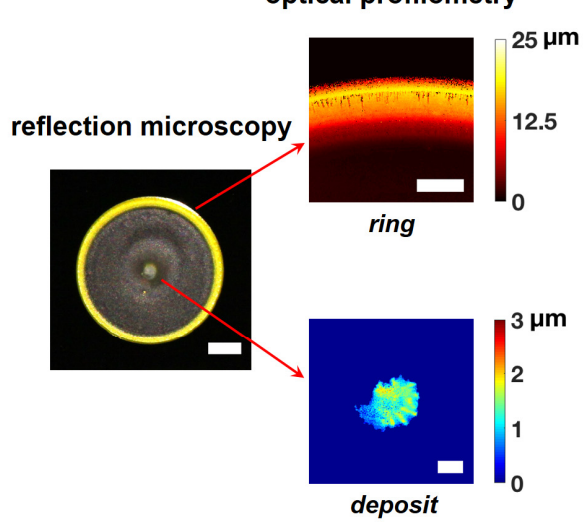

C

characterization of light-induced deposit
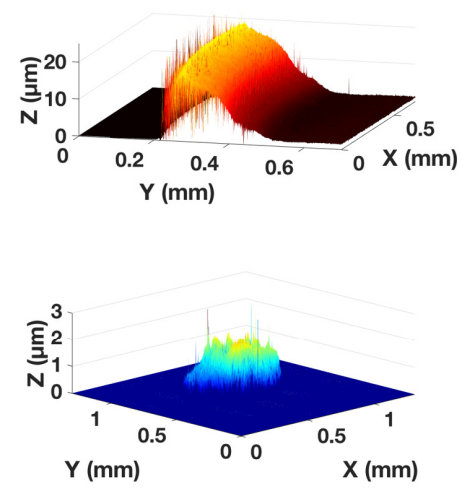

$\rightarrow$ radius \& height of deposit

Figure 1. Light-generated particle deposits from evaporating sessile drops of colloidal suspensions. a) Schematic of the experiment. A $7 \mu \mathrm{L}$ sessile drop containing AzoTAB cationic photosensitive surfactants (concentration $C_{s}$ ) and cationic polystyrene nanoparticles (NPs, $200 \mathrm{~nm}$ diameter, 
concentration $C_{p}$ ) is irradiated with a focused UV beam (radius $r_{U V}$, intensity $I_{U V}$ ) during drying. After complete evaporation, a light-induced central deposit of particles within an evaporation-driven "coffeering" pattern is obtained. b) Left: real color, low magnification reflection image of a typical final pattern obtained after the irradiation $\left(r_{U V}=84 \mu \mathrm{m}, I_{U V} \approx 260 \mathrm{~W} / \mathrm{m}^{2}\right)$ of an evaporating drop containing NPs $\left(C_{p}\right.$ $=4 \mathrm{mg} / \mathrm{mL})$ and AzoTAB $\left(C_{s}=10 \mathrm{mM}\right)$. The scale bar is $1 \mathrm{~mm}$. Right: Optical profilometry images of the ring pattern (top) and the light-directed central deposit (bottom). Scale bars are $200 \mu \mathrm{m}$. c) Threedimensional representations of the morphologies of the ring (top) and light-induced central particle deposit (bottom), as obtained from the optical profilometry data.

A reflection microscopy image of a typical deposit is shown in Figure 1b, left. Note that irradiation of smaller evaporating drops $(2 \mu \mathrm{L})$ yielded similar results, suggesting that eOMA is applicable regardless of the drop volume (Figure S1). Optical profilometry measurements quantitatively revealed the morphological structure and the geometrical characteristics of the two distinguishable regions comprising the composite pattern (Figures S2 and S3). At the drop edge (position of the pinned contact line), the emergence of the CRE ${ }^{13}$ affecting all non-volatile compounds of the suspension yielded a characteristic ring-shaped deposit made of NPs and AzoTAB ${ }^{42}$ (Figure 1b, top right). The width and the maximum height of the ring were about $300 \mu \mathrm{m}$ and $20 \mu \mathrm{m}$, respectively (Figure 1c, top). Notably, at the drop center (locus of the UV-exposed area), the light-induced flow pattern accumulated the particles into a cylinder-like deposit (Figure 1b, bottom right). Between these two zones, only a minor amount of materials was deposited despite the electrostatic interactions between the glass substrate and both particles and surfactants, showing the predominance of flows in controlling the deposition behaviour. We previously showed that, in eOMA, the Marangoni flows oriented toward the UV spot led to a recirculating flow pattern, which, in combination with the formation of Marangoni eddies at the edge of the drop, resulted in a volume of 'trapped' particles below the irradiated area. Although a significant amount of particles were brought to the contact line by the evaporation-driven flow, which became particularly strong at the end of 
the evaporation, a fraction of particles remained in this light-induced trapping volume until the last stages of evaporation to be deposited under the irradiated area. Notably, under these specific conditions $\left(C_{p}=4 \mathrm{mg} / \mathrm{mL} ; C_{s}=10 \mathrm{mM}, r_{U V}=84 \mu \mathrm{m} ; I_{U V} \approx 260 \mathrm{~W} / \mathrm{m}^{2}\right)$, the optical profilometry measurements showed that the deposit radius and median height were $213 \mu \mathrm{m}$ and $1.19 \mu \mathrm{m}$, respectively (Figure 1c, bottom). Several conclusions can be drawn at this stage. First, these results confirm that, despite the effective action of the evaporation-induced outward flow to bring NPs at the pinned contact line, a fraction of NPs were patterned at the irradiated location due to the action of the light-induced recirculation flow pattern. Second, the radial symmetry of the deposit was in agreement with the accumulation of particles controlled by the converging Marangoni flows but the deposit radius was significantly larger than that of the UV spot $\left(r_{U V}=84 \mu \mathrm{m}\right)$. Third, the upper surface of the deposit was rather flat with a thickness corresponding to a few layers of particles, showing that particles were deposited in a threedimensional fashion. All these results emphasize that, when assembling particles by eOMA, a concerted action of different effects should be at work to control the shape and size of the deposit. We thus focused on the geometrical properties of the central deposit and analyzed how it was affected by different parameters, to both better understand this deposition process and finely control of the geometry of individual UV-directed deposits.

We first hypothesized that by varying the diameter of the UV beam, the radial extent of the trapping volume and hence the number of accumulated NPs could be modulated, allowing us to tune the number of NPs assembled in the central deposit. To test this hypothesis, we kept the UV intensity constant at $I_{U V} \approx 260 \mathrm{~W} / \mathrm{m}^{2}$ (to maintain fixed the optical Marangoni flow) and we varied the radius of the UV spot, $r U V$. Figure 2a shows optical profilometry images of patterns formed after the irradiation of drops containing a fixed amount of polystyrene NPs $\left(C_{p}=2 \mathrm{mg} / \mathrm{mL}\right)$ and $\operatorname{AzoTAB}\left(C_{s}=10 \mathrm{mM}\right)$. 
a

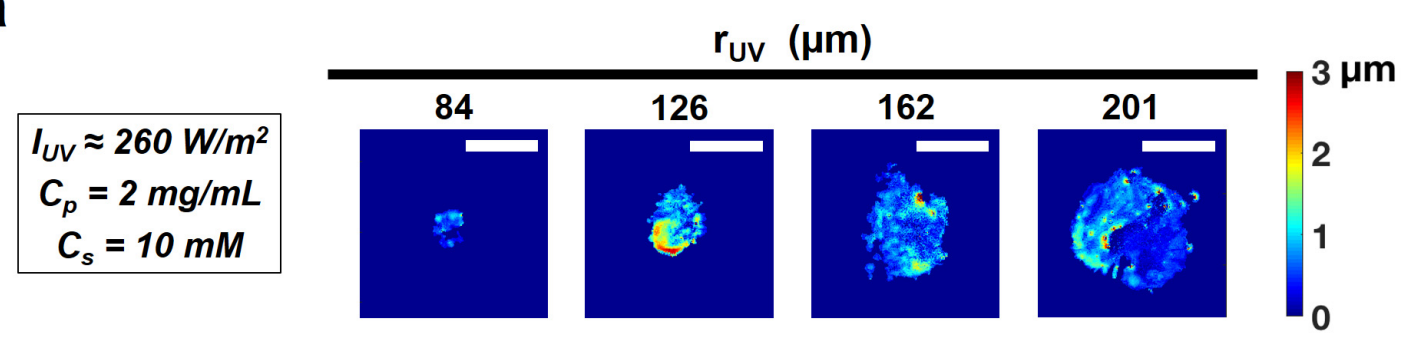

b

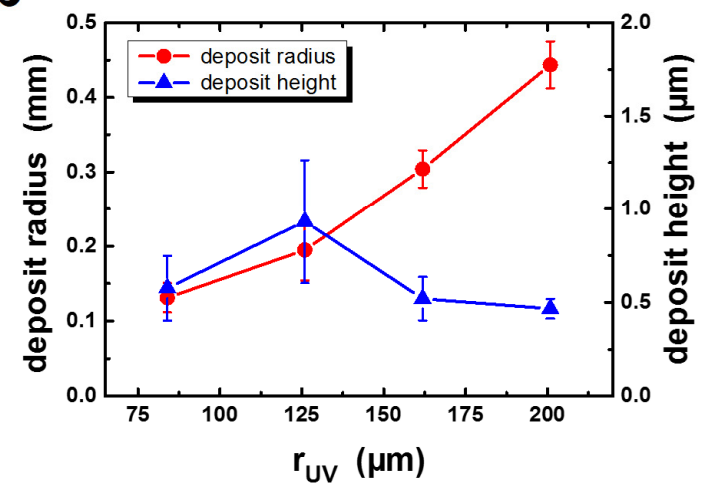

C

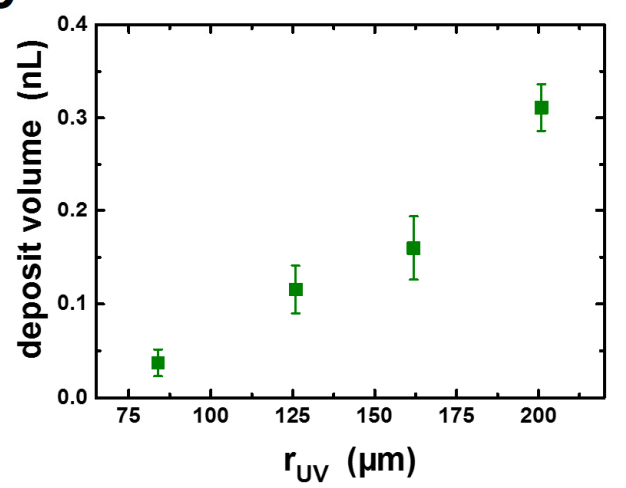

Figure 2. The size of the UV beam controls the lateral extent of the light-induced central particle deposit. (a) Optical profilometry images of light-generated particle deposits obtained using UV beams of different radii, $r_{U V}$; the UV intensity was kept constant $\left(I_{U V} \approx 260 \mathrm{~W} / \mathrm{m}^{2}\right)$. All scale bars are $500 \mu \mathrm{m}$. b) The radius and median height of the central deposits as a function of $r_{U V} . \mathrm{c}$ ) The volume of the central deposit versus $r_{U V}$. Symbols and error bars represent mean values and their standard deviations, respectively, obtained from measurements of four independent deposits.

Low magnification reflection microscopy images of the same patterns are shown in Figure S4. Figure 2a clearly shows that the size of the light-directed NP deposits continuously increased with increasing beam diameter. Image analysis of the height profiles revealed that an increase of $r_{U V}$ from $84 \mu \mathrm{m}$ to $201 \mu \mathrm{m}$ resulted in an increase in deposit radius from $131 \pm 20$ to $444 \pm$ $31 \mu \mathrm{m}$. At the same time, the mean height of the deposits was almost insensitive to the size of the UV spot, with a constant value of approximately $0.6 \mu \mathrm{m}$ (Figure $2 \mathrm{~b}$ ). These dependencies of the characteristic size of the dry patterns on the beam size, led to a continuous increase of the volume of the deposited NPs as a function of the beam radius (Figure 2c). The minimum 
beam radius, $r U V=84 \mu \mathrm{m}$ led to the formation of a deposit with a volume of $0.037 \pm 0.014 \mathrm{~nL}$, whereas the largest beam utilized, $r U V=201 \mu \mathrm{m}$ resulted in the maximum deposit volume equal to $0.311 \pm 0.025 \mathrm{~nL}$. These results demonstrate that the number of particles assembled in the central deposit increased with the size of the UV beam. The deposit height was almost constant leading to radii approximately twice the radius of the UV beam.

Next, we hypothesized that an alternative way to control the number of NPs gathered within the trapping volume and ultimately the deposition efficiency, was to vary the UV intensity. For a constant beam diameter, increasing $I_{U V}$ should result in both a larger spatial flow extent and a stronger flow speed ${ }^{39}$, that is, a larger number of optically accumulated particles. We tested this hypothesis by maintaining the beam radius fixed at $r_{U V}=84 \mu \mathrm{m}$ and conducting eOMA experiments with different $I U V$. We used drops of fixed composition $\left(C_{p}=2 \mathrm{mg} / \mathrm{mL}\right.$ and $C_{S}=$ $10 \mathrm{mM}$ ). Increasing the number of photons impinging on the free surface of the drop led to NP deposits with increasing diameter, as seen from the optical profilometry (Figure 3a) as well as the reflection microscopy (Figure S5) images. The deposit radius was found to monotonously increase with an increase in the UV intensity (Figure 3b). The pattern corresponding to the minimum intensity used here $\left(I_{U V}, \min =260 \mathrm{~W} / \mathrm{m}^{2}\right)$ displayed a radius of $131 \pm 20 \mu \mathrm{m}$. Increasing $I_{U V}$ by a factor of $5.7\left(I_{U V, \max }=1490 \mathrm{~W} / \mathrm{m}^{2}\right)$, yielded a NP deposit with a radius that increased by a factor of 2.6 (maximum radius of $337 \pm 28 \mu \mathrm{m}$ ). Conversely, the median height of the deposit did not show a clear trend with the UV intensity and seemed to be roughly constant, with an average value of $0.704 \pm 0.194 \mu \mathrm{m}$ (Figure 3b). Accordingly, the volume of the deposits increased with the UV intensity, reaching a value of $0.205 \pm 0.073 \mathrm{~nL}$ at the highest intensity utilized in this study $\left(I_{U V}, \max =1490 \mathrm{~W} / \mathrm{m}^{2}\right)$. These findings indicate that both the lateral dimension of the UV-directed deposits and the number of particles contained within (i.e., the deposition efficiency) could be modulated by simply varying the UV intensity, the deposit height remaining almost unaffected. 
a

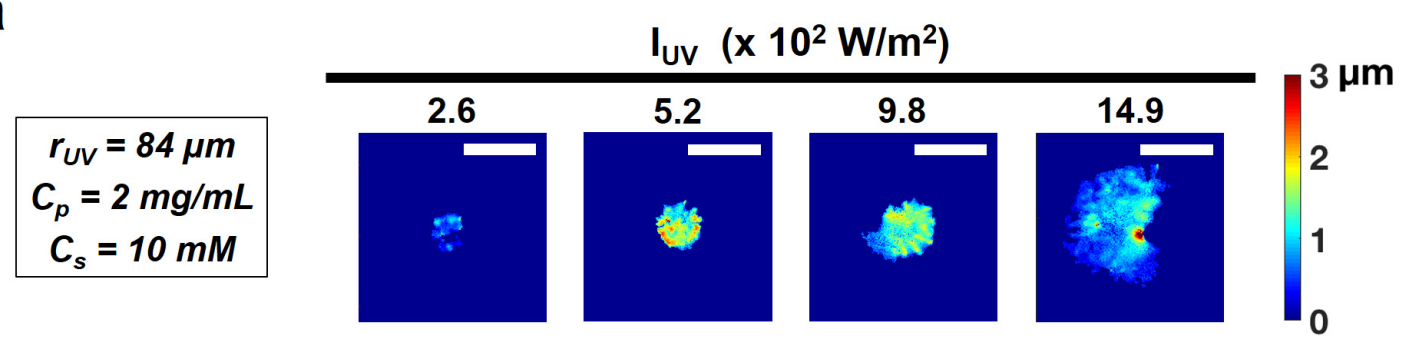

b

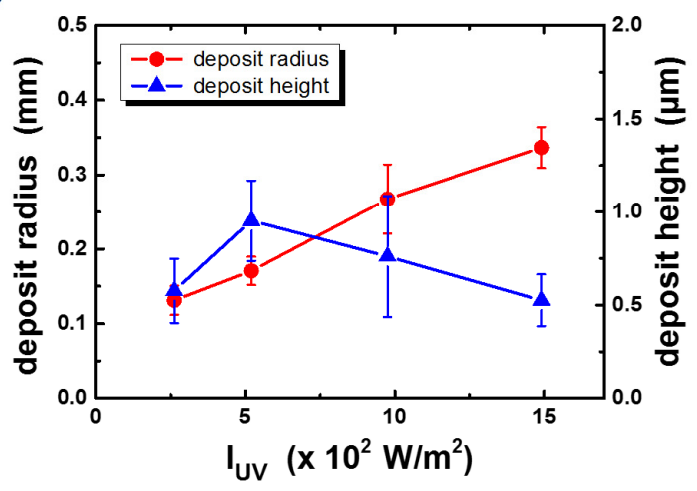

C

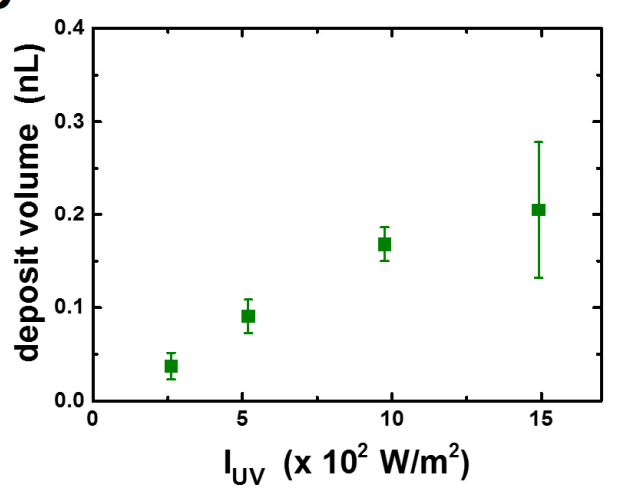

Figure 3. The UV intensity controls the lateral size of the light-induced central particle deposit. a) Optical profilometry images of light-driven particle deposits obtained with varying UV light intensity $I_{U V}$; the beam radius was fixed at $r_{U V}=84 \mu \mathrm{m}$. All scale bars are $500 \mu \mathrm{m}$. b) The radius and median height of the central deposits as a function of $I_{U V}$. c) The volume of the central deposit versus $I_{U V}$. Symbols and error bars represent mean values and their standard deviations, respectively, corresponding to four independent deposits.

Since the deposit characteristics were shown to be directly affected by the strength of the optical Marangoni flows, we also explored non-optical ways to tune these surface flows. In the presence of a surface tension gradient $\nabla \gamma$, the speed of the Marangoni flow at the liquid surface is $U_{M a} \sim(h / \eta) \cdot \nabla \gamma$, where $h$ is the height of the liquid and $\eta$ the dynamic viscosity. ${ }^{41}$ We expected that $\nabla \gamma$ should depend on the initial AzoTAB concentration in the drop, $C_{s}$, and therefore we then studied how $C_{s}$ affected the deposition behaviour. To quantitatively explore the influence of $C_{s}$ on both the deposition efficiency and the morphology of the deposited NPs, 
we fixed the optical parameters $\left(I_{U V} \approx 260 \mathrm{~W} / \mathrm{m}^{2}, r_{U V}=84 \mu \mathrm{m}\right)$ and we kept the NP concentration constant $\left(C_{p}=2 \mathrm{mg} / \mathrm{mL}\right)$. When drops with a low AzoTAB concentration $\left(C_{s}=\right.$ $1 \mathrm{mM}$ ) were irradiated by UV light, a disk-shaped deposit was obtained, with the NPs being uniformly distributed over the whole (initially) wetted area (Figure S7). Surprisingly, when samples with even lower $C_{s}(0.5 \mathrm{mM})$ were dried, patterns displaying a homogeneous disk-like morphology were observed in drops that were either exposed to or protected from UV light. This unexpected result might be attributed to the action of Marangoni eddies that develop in evaporating drops containing surfactants. ${ }^{45}$ Conversely, at the maximum AzoTAB amount used here $\left(C_{s}=13.5 \mathrm{mM}\right)$, which was close to the critical micellar concentration $(\mathrm{CMC}=12.6 \mathrm{mM}$ and $14.5 \mathrm{mM}$ for trans- and cis-AzoTAB ${ }^{46}$ ), we could not detect any significant amount of NP being deposited in the drop center. We thus focused our quantitative analysis of the deposit characteristics for $C_{s}$ ranging from 2.5 to $10 \mathrm{mM}$ and found that the lateral dimension of the light-generated NP pattern decreased with an increase in $C_{s}$ (Figure 4). Figure $4 \mathrm{~b}$ shows the evolution of the radius of the central deposit with $C_{s}$. For $C_{s}=2.5 \mathrm{mM}$, we observed the formation of the largest NP pattern in the experiments reported in this study (Figures 4a, S6 and Movie S1) with a radius $667 \pm 160 \mu \mathrm{m}$. A sharp decrease to $247 \pm 65 \mu \mathrm{m}$ was observed at $C_{s}=5 \mathrm{mM}$ followed by a smoother decrease to $131 \pm 20 \mu \mathrm{m}$ that remained approximately constant for $\mathrm{C}_{\mathrm{s}}=7.5 \mathrm{mM}$ and $10 \mathrm{mM}$. Similarly to its dependence on the optical parameters, the median height of the central pattern did not vary with $C_{s}$, displaying an almost constant value of $0.644 \pm 0.086 \mu \mathrm{m}$. The volume occupied by the NPs deposited within the central pattern showed a similar evolution with $C_{s}$, reaching a maximum value of $1.149 \pm 0.452 \mathrm{~nL}$ for $C_{s}=2.5 \mathrm{mM}$ (Figure $4 \mathrm{c}$ ). This corresponded to the maximum light-directed deposition efficiency observed in our experiments. The reported findings indicated that varying the AzoTAB concentration provided a non-optical pathway for modulating both the radial 
a

dimension of the patterns and the number of NPs deposited within. Notably a large number of NPs were trapped and finally deposited at a low AzoTAB concentration.

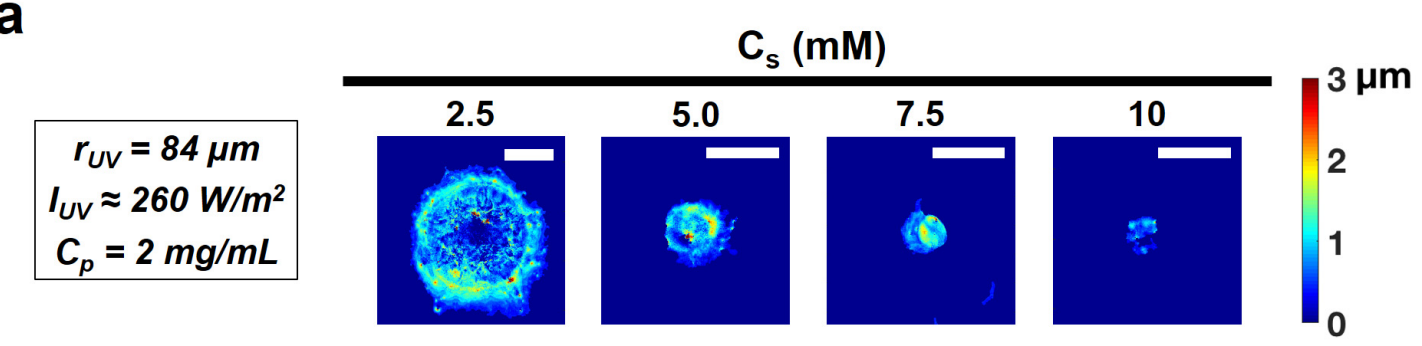

b

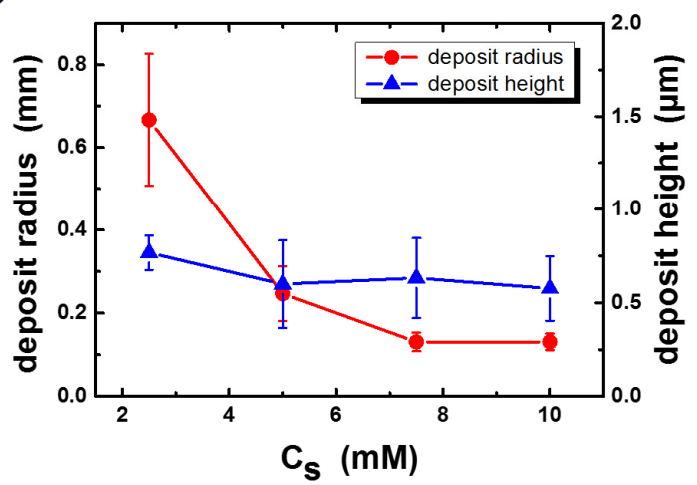

C

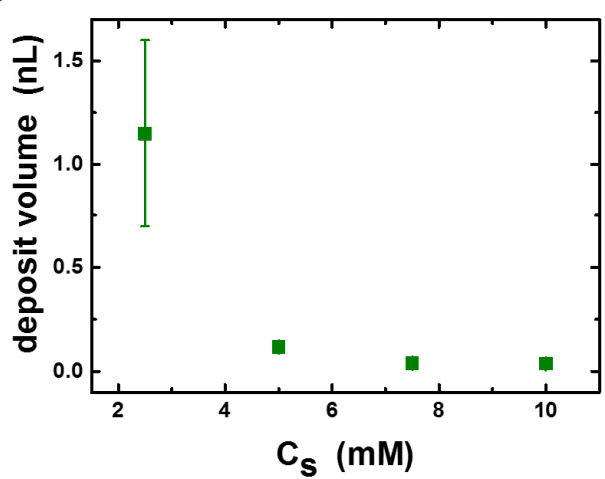

Figure 4. Controlling the lateral extent of the light-driven central particle deposit by adjusting the concentration of photosensitive surfactants in the suspension drop. The beam radius and intensity were kept constant $\left(r_{U V}=84 \mu \mathrm{m}\right.$ and $I_{U V} \approx 260 \mathrm{~W} / \mathrm{m}^{2}$, respectively). a) Optical profilometry images of lightdirected particle deposits obtained with varying UV light intensity. All scale bars are $500 \mu \mathrm{m} . \mathrm{b}$ ) The central deposit radius and height as a function of AzoTAB concentration $C_{s}$. c) The central deposit volume versus $C_{s}$. Symbols and error bars represent mean values and their standard deviations, respectively, obtained from measurements of four independent deposits.

To shed more light on the strong dependence of NP patterning on AzoTAB concentration, we investigated the surface tension $(\gamma)$ of drops of aqueous AzoTAB solutions that were irradiated under similar conditions to the ones in a typical eOMA experiment (see Experimental section for details). Under homogeneous white light illumination, the surface tension decreased with time for $C_{s}=2.5-13.5 \mathrm{mM}$, displaying a similar kinetics due to 
surfactant adsorption, while it remained almost constant for the lowest concentration $\left(C_{s}=\right.$ $1 \mathrm{mM}$ ) (Figure S8). Switching on the UV light resulted in a rise of the surface tension for all AzoTAB concentrations, which reached a plateau value at longer times. However, the kinetics of the $\gamma$ increase as well as the time required to reach the plateau, markedly depended on $C_{s}$ (Figure S8). Figure 5a shows the evolution of the surface tension difference $\Delta \gamma$ between the plateau value $\left(\gamma_{W+U V}\right)$ and the value immediately prior to switching the UV light on $\left(\gamma_{W}\right)$, as a function of $C_{s}$. Interestingly, a non-monotonic $\Delta \gamma\left(C_{s}\right)$ dependence was observed. Upon increasing $C_{s}, \Delta \gamma$ strongly increased from its minimum of $\Delta \gamma=0.5 \pm 0.4 \mathrm{mN} / \mathrm{m}$ for $C_{s}=1 \mathrm{mM}$ to a maximum value of $\Delta \gamma=11.8 \pm 1.6 \mathrm{mN} / \mathrm{m}$ for $C_{s}=7.5 \mathrm{mM}$. Increasing $C_{s}$ further led to a steep $\Delta \gamma$ decrease, which reached $\Delta \gamma=1.5 \pm 0.7 \mathrm{mN} / \mathrm{m}$ for $C_{s} \approx C M C=13.5 \mathrm{mM}$. Note that the reported $\Delta \gamma\left(C_{s}\right)$ evolution holds for pendant drops of an almost fixed AzoTAB concentration. In a drying sessile drop, the surfactant concentration continuously increases from its initial value $C_{s}$ as water molecules keep evaporating. Hence, for the sake of simplicity, we may assume that the time evolution of the light-induced surface tension difference $\Delta \gamma$ has a qualitatively similar shape to the experimental $\Delta \gamma\left(C_{S}\right)$ curve shown in Figure 5a. This led us to consider the hypothetic situation of three sessile drops, irradiated in the same manner and containing a fixed amount of NPs and a low $\left(C_{s}<<C M C\right)$, intermediate $\left(C_{s}<C M C\right.$ and corresponding to the maximum of $\Delta \gamma)$ and high $\left(C_{s} \approx C M C\right)$ initial AzoTAB concentration, respectively. As previously mentioned, the number of particles trapped by the recirculating optical Marangoni flow should be directly correlated to both the strength and the spatial extent of the flow. Given that the optical characteristics of the irradiation are fixed, we can thus assume that the flow strength and thus the number of trapped particles will be directly linked to the surface tension gradient at the drop surface $\nabla \gamma$ integrated over the evaporation time. Since the surface tension in and out of the UV irradiated area can be assimilated to $\gamma_{W+U V}$ and $\gamma_{W}$, respectively, we can assume $\nabla \gamma(t)$ to be proportional to $\Delta \gamma(t)$ and therefore to $\Delta \gamma\left(C_{S}\right)$ 
(Figure 5b). As a consequence, for $C_{s}<<C M C, \nabla \gamma$ will remain significant for most of the evaporation time of the drop, leading to a large number of trapped particle and a strong assembly efficiency (Figure 5b, orange curve). For $C_{s}<C M C, \nabla \gamma$ will start from the strongest value but it will decrease as evaporation evolves and it will finally vanish when $C_{s}$ reaches $\mathrm{CMC}$, leading to a moderate number of trapped particles (blue curve). Finally, for $C_{s} \approx C M C$, $\nabla \gamma$ will be almost zero during the whole evaporation time, leading to the lowest NP accumulation within the trapping zone and a poor deposition efficiency (green curve). Note that in this simple explanation, we did not take into account that the evaporation-driven outward flow also increases during the evaporation process. If the initial concentration of surfactants is too low, the maximal $\nabla \gamma$ will be reached only in the very late stage of evaporation, i.e., when the evaporation-driven outward flow becomes also very strong. Note that we did not observe such a regime in our explored surfactant concentrations $\left(C_{s} \geq 0.5 \mathrm{mM}\right)$ except in the situation without surfactant where no particles could be deposited under the UV beam.

a

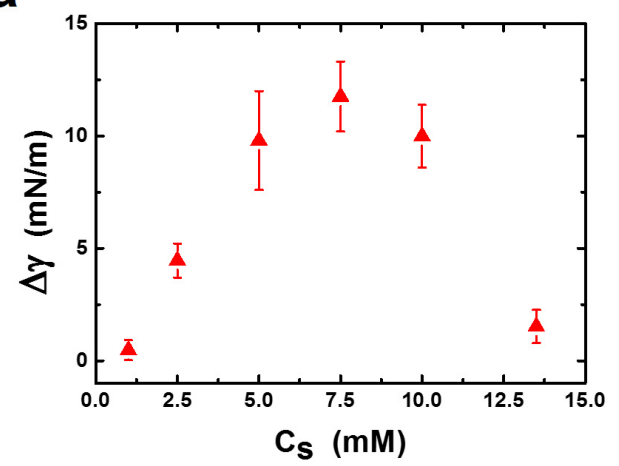

b

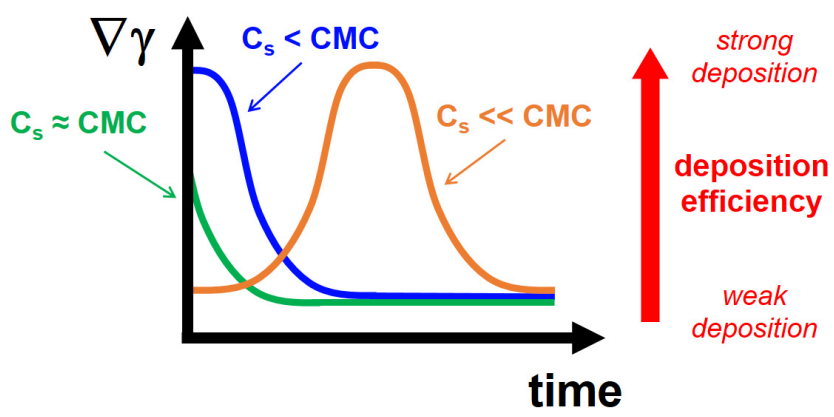

Figure 5. The UV-induced surface tension alteration and its effect on the patterning mechanism. a) The difference $\Delta \gamma=\gamma_{W+U V}-\gamma_{W}$, between respectively the surface tension of an aqueous AzoTAB solution drop upon visible light irradiation immediately prior to switching the UV light on $\left(\gamma_{W}\right)$, and the plateau value of the surface tension under simultaneous white and UV irradiation $\left(\gamma_{W+U V}\right)$, versus the AzoTAB concentration $C_{s}$. b) Schematic representation of the temporal evolution of the 
surface tension gradient at the drop surface $(\nabla \gamma)$ during the light-directed deposition process and its influence on NP deposition efficiency. For drops of low initial AzoTAB concentration $\left(C_{s}<<C M C\right)$, NPs are gathered within the trapping zone for a maximum accumulation time due to the long time required to reach the $C M C$ during drop drying. This results in the maximum number of deposited NPs after complete evaporation. For intermediate AzoTAB concentrations corresponding to the maximum of $\Delta \gamma$ in Figure $5 \mathrm{a}\left(C_{s}<C M C\right)$, the trapping efficiency is strong at the beginning but rapidly vanishes with time due to the decrease of $\nabla \gamma$, leading to moderate deposition efficiency. For $\left.C_{s} \approx C M C\right) \nabla \gamma$ is very small for the whole drop evaporation and only a small number of particles are deposited at the UVirradiated area.

All results presented above showed that, in eOMA, both the volume and the radius of the individual central deposit could be controlled by the parameters of the optical stimulation (size and intensity), or by the concentration of photosensitive surfactants. The general trend was that stronger Marangoni flows over the course of evaporation led to a stronger particle deposition efficiency and a larger volume of the final deposit. Surprisingly, for a fixed particle concentration, most of the deposits displayed a flat geometry and a similar thickness (around $0.6 \mu \mathrm{m})$ regardless of irradiation properties and AzoTAB concentration. The most remarkable feature of the deposits was that their thickness corresponded to a few layers of particles. We can thus propose that two mechanisms are at work in controlling the geometry of the deposit. On the one hand, the parameters mentioned above (irradiation properties, AzoTAB concentration), via their roles in tuning the trapping efficiency by controlling the strength of the recirculating optical Marangoni flow, determine the number of deposited particles, i.e., the volume of the deposit. On the other hand, an independent sweeping mechanism at the very late stage of evaporation should exist on already deposited or depositing particles to flatten the deposit until a given thickness. Assuming that the volume of the assembled particles does not change much in this process, the radius of the deposit varies accordingly. We suggest the 
evaporation-driven outward flow at the very late stage of evaporation, which is strong and mainly parallel to the substrate, to be mainly involved in this sweeping mechanism that controls the final deposit thickness.

To test the above-proposed mechanism and to seek for a way to control the vertical dimension of the deposit, we varied the particle concentration. We hypothesized that increasing the number of NPs within the drop should lead to deposits with both larger volume and larger heights. Our hypothesis was based on the fact that the viscosity of the drop increases with increasing evaporation time, this increase being stronger for drops with higher initial particle volume fractions. Given that an increased viscosity acts against the transport of NPs to the drop edge due to the action of the outward capillary flow, ${ }^{17}$ we expected the volume of the patterned NPs to increase. This increase in viscosity should also weaken the spreading of NPs at the end of the drying process, thus leading to deposits of increased height. Figure 6a shows the threedimensional morphology of deposits formed after the drying of UV-irradiated drops with different initial NP concentrations $C_{p}$ (reflection microscopy images of these deposits are shown in Figure S9). The optical parameters as well as the initial AzoTAB concentration were fixed $\left(r U V=84 \mu \mathrm{m}, I_{U V} \approx 260 \mathrm{~W} / \mathrm{m}^{2}, C_{S}=10 \mathrm{mM}\right)$. A clear increase of both the radial and vertical extent of the deposit was observed. Increasing $C_{p}$ from 2 to $6 \mathrm{mg} / \mathrm{mL}$ led to an increase of the radius and the mean height of the deposit from $131 \pm 20$ and $0.576 \pm 0.160 \mu \mathrm{m}$ to $249 \pm$ 26 and $1.201 \pm 0.203 \mu \mathrm{m}$, respectively (Figure $6 \mathrm{~b}$ ). The evolution of the volume of the lightinduced patterns with $C_{p}$ exhibited a similar evolution to the one of the pattern dimensions, reaching a maximum value of $0.264 \pm 0.064 \mathrm{~nL}$ for the highest NP concentration $\left(C_{p}=6\right.$ $\mathrm{mg} / \mathrm{mL}$ ) used (Figure 6c). Moreover, the deposits at the highest NP concentration exhibited a more rounded upper surface, in agreement with a less efficient sweeping mechanism at the late stage of evaporation. The above results demonstrate that in eOMA, varying the initial NP 
content within the sessile drop provides a straightforward way to adjust the three-dimensional morphology of the dry NP pattern.

a

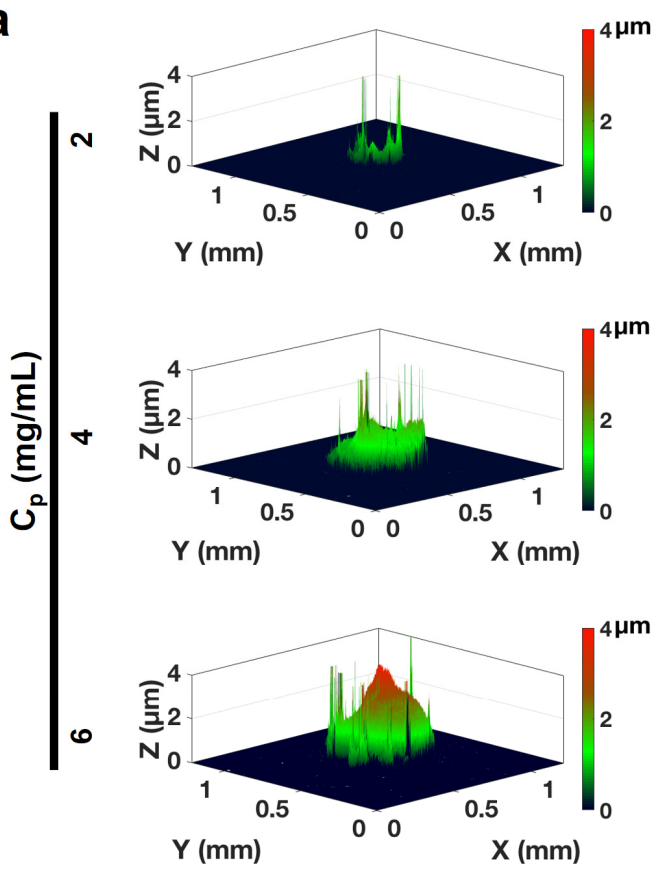

b

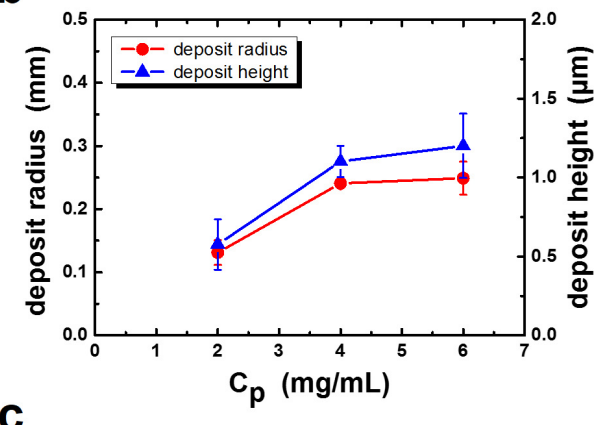

C

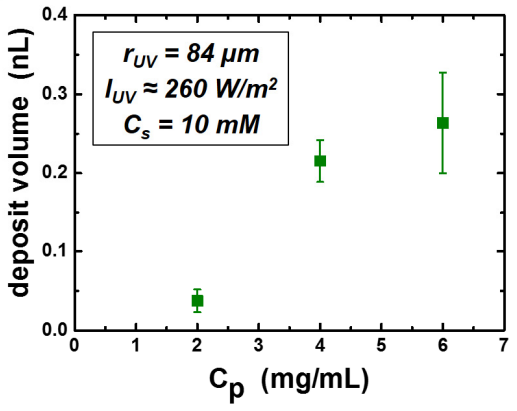

Figure 6. Tuning the lateral and the vertical dimensions of the light-directed central particle deposit by adjusting the particle concentration in the suspension drop. The beam radius and intensity were fixed at $r_{U V}=84 \mu \mathrm{m}$ and $I_{U V} \approx 260 \mathrm{~W} / \mathrm{m}^{2}$, respectively. a) Three-dimensional representations of the optical profilometry data of light-generated particle deposits formed from drops of suspensions of varying NP concentration $C_{p}$. b) The central deposit radius and height as a function of $C_{p}$. c) The central deposit volume versus $C_{p}$. Symbols and error bars represent mean values and their standard deviations, respectively, corresponding to four independent deposits.

After studying how to control the deposit geometry through a rational adjustment of different control parameters, we investigated the robustness of eOMA by checking its applicability to other configurations. To investigate the influence of the properties of the solid 
substrate, we placed water drops containing polystyrene NPs (diameter $200 \mathrm{~nm}, C_{p}=2 \mathrm{mg} / \mathrm{mL}$ ) and AzoTAB $\left(C_{s}=10 \mathrm{mM}\right)$ on thin vinyl sheets, instead of the glass coverslips used for all previous experiments. Whereas the wettability of this substrate is lower than the glass coverslips (initial contact angles of about $58^{\circ}$ and $71^{\circ}$ for the drops on glass and vinyl substrates, respectively, in the experiments shown in Figure S10), the contact line of the sessile drop remained pinned during evaporation, which is a prerequisite for effective patterning. ${ }^{42}$ In the absence of UV light, the dry pattern showed a typical coffee-ring morphology (Figure S10). Illumination of the sessile drop with UV light $\left(r_{U V}=126 \mu \mathrm{m}, I_{U V} \approx 260 \mathrm{~W} / \mathrm{m}^{2}\right)$ led to a central deposit of radius of about $220 \mu \mathrm{m}$ (Figure S10b). The characteristics of the light-generated deposit on the vinyl substrate were very similar to the ones corresponding to a patterned created on glass (Figure S10c). These findings demonstrate that eOMA can be successfully employed to pattern NPs on solids of different surface chemistries and wettabilities, the only requirement being the firm pinning of the contact line on the substrate.

Finally, we explored the possibility of using light to pattern much smaller NPs compared to what we had previously achieved (minimal diameter was $200 \mathrm{~nm}),{ }^{42}$ as many of the properties of nanoparticles are emphasized when their size decreases. ${ }^{1}$ This represents a patterning challenge as reducing the NP dimensions enhances the action of Brownian diffusion, which acts against the accumulation of NPs under the irradiated area. For these experiments, we employed aqueous sessile drops containing cationically modified silica NPs (diameter 30 $\left.\mathrm{nm}, C_{p}=10 \mathrm{mg} / \mathrm{mL}\right)$ and AzoTAB $\left(C_{s}=10 \mathrm{mM}\right)$. In the absence of UV light, a coffee-ring pattern encircling a significant amount of deposited silica NPs was observed after complete evaporation. These NPs were scattered over an extended area around the drop center, forming an approximately circular central deposit of radius $\sim 460 \mu \mathrm{m}$ (Figure 7a). Irradiating the center of the sessile drop with UV light $\left(r_{U V}=126 \mu \mathrm{m}, I_{U V}=980 \mathrm{~W} / \mathrm{m}^{2}\right)$ resulted in a qualitatively different dry deposit: similarly to the control experiment (Figure 7a), a ring-shaped pattern was 
formed, however it encircled a dense circular NP deposit at the drop center (Figure 7b). This deposit had a radius of $\sim 140 \mu \mathrm{m}$, very close to the size of the UV beam used. To further confirm that the clear accumulation of NPs was the result of UV light, we performed another experiment, where an off-center UV irradiation was applied on the drop (Figure 7c). After drop evaporation, a NP deposit with geometrical features very similar to the in-center experiment was created exactly at the locus of the UV beam. The above findings clearly demonstrate that eOMA can be successfully employed to accurately pattern small colloids despite the strong counter-acting Brownian motion.

a
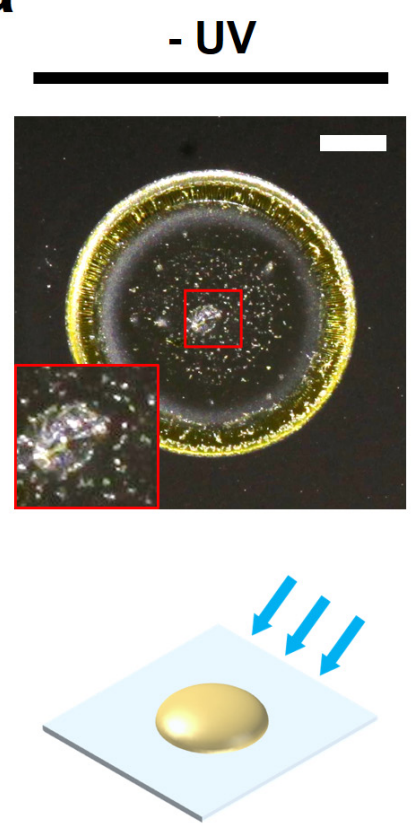

b
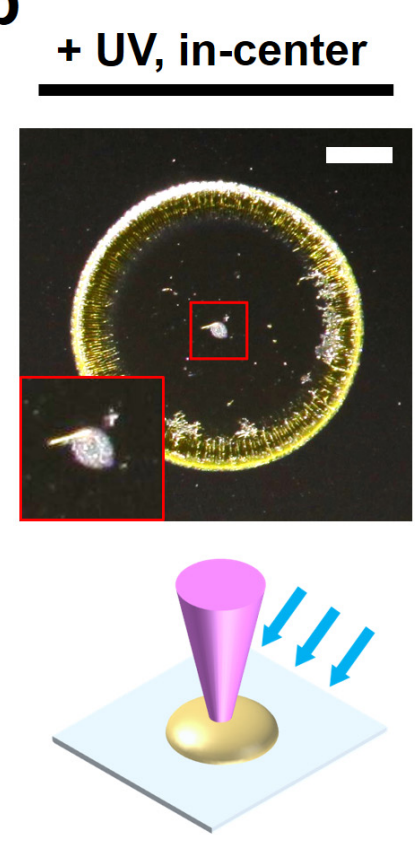

C
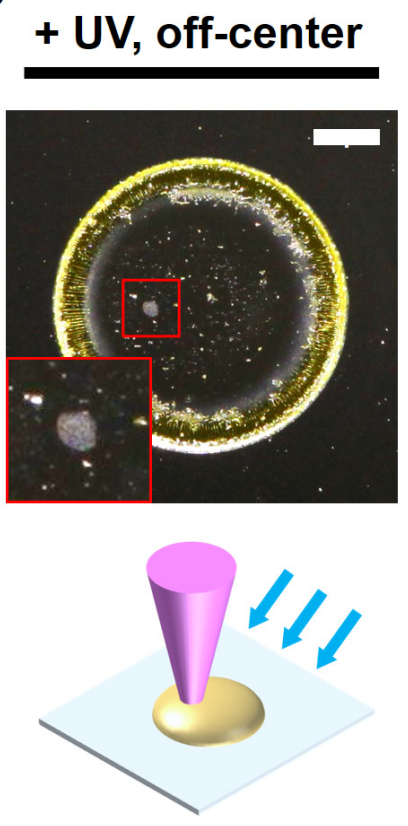

Figure 7. Patterning cationic silica NPs of diameters down to $30 \mathrm{~nm}$ in diameter by eOMA. Real color reflection microscopy images of the patterns obtained after the complete evaporation of $7 \mu \mathrm{L}$ drops $\left(C_{p}=10 \mathrm{mg} / \mathrm{mL}, C_{s}=10 \mathrm{mM}\right)$, exposed to different light conditions. a) Drop drying in the absence of UV light (control experiment). b) Evaporation of a drop, the center of which was exposed to the UV beam $\left(r_{U V}=126 \mu \mathrm{m}, I_{U V}=980 \mathrm{~W} / \mathrm{m}^{2}\right)$. c) Evaporation of a drop which was irradiated with an off- 


\section{CONCLUSIONS}

In summary, we employed optical profilometry measurements and image analysis to quantitatively investigate the geometrical characteristics of colloidal deposits formed via eOMA in sessile drops containing NPs and AzoTAB. First, we showed that by increasing the intensity or the diameter of the UV beam, the radial dimension of the deposits was increased, whereas their height remained almost constant. The combination of these effects led to an increased number of deposited NPs at the UV-irradiated area. Second, a decrease in AzoTAB concentration yielded deposits of increased diameter and a constant height, thus corresponding to a larger volume of deposited particles. Third, both the height as well as the diameter (and thus the volume) of the deposits were controlled by varying the NP concentration of the suspensions. These dependencies were explained on the basis of qualitative considerations that related the deposition efficiency (experimentally expressed by the total volume of the lightcontrolled deposited particles) to the geometrical extent and the speed of the optical Marangoni flows. All these findings show that a rational choice of the optical or material parameters involved in the eOMA process can reproducibly result in NP deposits, the three-dimensional geometrical characteristics of which are accurately tunable.

Furthermore, in addition to glass substrates, we have shown that NPs can be deposited at the locus of the irradiated area on more hydrophobic polymeric substrates as well. The only requirement for effective patterning is the firm pinning of the three phase contact line on the used substrate, for most of the drop lifetime. This result suggests that colloids can be precisely placed via eOMA on a plethora of different solid surfaces, regardless of their specific wetting 
and chemical properties (provided that contact line pinning occurs), confirming the versatility and robustness of this patterning method.

Finally, we achieved to pattern small silica NPs (diameter $30 \mathrm{~nm}$ ) on glass substrates. To the best of our knowledge, this is the minimum particle size to be patterned solely by lightinduced flows in an evaporating drop. This finding suggests that UV-driven optical Marangoni flows within the drops can largely overcome both the diffusive motion (becoming stronger with decreasing particle size) and the evaporation-driven outward convective flow. As a result, eOMA appears to be a powerful tool to direct the deposition of various kinds of particles within a size range spanning at least three orders of magnitude (tens of $\mathrm{nm}$ to $\mu \mathrm{m}$ ).

We foresee that the precise control of the features of an individual NP deposit presented here and the previously reported capability of creating arbitrarily-shaped and/or multiple deposits utilizing structured UV light, ${ }^{42}$ will open a new route to precise and complex particle patterning. Combined with its versatility in terms of particle and substrate types, eOMA thus appears as a new valuable tool for robust and reconfigurable light-directed particle patterning.

\section{EXPERIMENTAL SECTION}

Materials. Ultrapure water (resistivity $18.2 \mathrm{M} \Omega \cdot \mathrm{cm}$ ) was used for all experiments. Azobenzene trimethylammonium bromide surfactant, AzoTAB, was synthesized as previously described. ${ }^{46}$ Its critical micellar concentration is $C M C_{\text {trans }}=12.6 \mathrm{mM}$ and $C M C_{\text {cis }}=14.6 \mathrm{mM}$ for the trans and the cis isomer, respectively. ${ }^{46}$ Fluorescent amine-modified polystyrene particles (diameter $200 \mathrm{~nm}$ ) were purchased from Thermo Fisher Scientific (Catalogue Number F8764, Lot Number 1503580). Triethoxylpropylaminosilane functionalized silica NPs 
(diameter $30 \mathrm{~nm}$ ) were purchased from Sigma-Aldrich (Product Number: 791342, Batch Number: MKBR3999V).

Preparation of colloidal suspensions. For preparing the suspensions, the appropriate volumes of water, stock AzoTAB aqueous solution $(25 \mathrm{mM})$ and stock particle suspension $\left(10 \mathrm{mg} \cdot \mathrm{mL}^{-}\right.$ ${ }^{1}$ for polystyrene particles; $40 \mathrm{mg} \cdot \mathrm{mL}^{-1}$ for silica NPs) were added in an Eppendorf tube. After mixing with a micropipette (Eppendorf), the samples were sonicated for $1 \mathrm{~min}$ in an ultrasonic bath and were stored under ambient light conditions.

Drop deposition. Prior to each experiment, the suspension was vortexed for about $1 \mathrm{~min}$, sonicated for $3 \mathrm{~min}$ and vortexed again for $1 \mathrm{~min}$. A drop (typical volume $7 \mu \mathrm{L}$, except otherwise stated) was then immediately deposited on the substrate by means of a micropipette. For all experiments but the one showed in Figure 6, substrates were thin glass coverslips (Menzel-Gläser), used as received. We have measured a static contact angle of $\theta=56.8 \pm 4.7^{\circ}$ for sessile drops of ultrapure water (in air) on the utilized substrates. ${ }^{23}$ For the experiment shown in Figure 6, a more hydrophobic vinyl substrate was used (Dominique Dutscher, Catalogue Number: 052951), which was successively swept with water- and ethanol-wet tissues prior to drop deposition. All experiments were carried out at a temperature and relative humidity range of $22.0 \pm 0.5{ }^{\circ} \mathrm{C}$ and $45.8 \pm 7.3 \%$, respectively.

Drop irradiation and drying. For drop irradiation, we utilized an optical setup similar to the one used earlier. ${ }^{42}$ Briefly, the divergent beam from an LED UV source (wavelength $370 \mathrm{~nm}$, maximum power 1.2 W, Intelligent LED Solutions) was first collimated with an appropriate set of lenses and was then focused by an objective (NA=0.13, Thorlabs). The UV beam was focused at the substrate surface $(84 \mu \mathrm{m})$, and the suspension drop was positioned in such a way that UV beam passed through the center of the drop. The UV power was adjusted by means of appropriate neutral density filters. A maximum power of about $33 \mu \mathrm{W}$ was obtained (measured 
after the focussing objective), which was modulated to $21.6,11$ and $5.8 \mu \mathrm{W}$ using neutral density filters having optical density $0.1,0.2$ and 0.5 respectively. The UV beam size was tuned by varying the vertical position of (i.e., defocusing) the objective, which was mounted to a translation stage. We employed four different spot radii in our study: $84 \mu \mathrm{m}, 126 \mu \mathrm{m}, 162 \mu \mathrm{m}$ and $201 \mu \mathrm{m}$. Simultaneously to UV irradiation, white light from an LED array was uniformly shined on the whole drop.

The substrate holding the deposited drop was mounted to the stage of an inverted fluorescent microscope (Axio Observer Z1, Zeiss). During irradiation and evaporation, a Petri dish cap with an optical window made from a glass coverslip was placed over the drop, to partially protect it by contamination and air currents.

Imaging of the light-directed deposits. Fluorescent images (and videos) during the patterning process, as well as images of the final (dry) deposits were acquired by means of an EMCCD camera (Evolve, Photometrics), attached to the microscope. Real-color reflection images of the deposited patterns were captured with a digital reflex camera (EOS 5D Mark II, Canon) equipped with a macro lens (EF $100 \mathrm{~mm}$, f 1:2.8, $67 \mathrm{~mm}$ diameter, Canon). White light illumination (at an angle of about $30^{\circ}$ with respect to the optical table, parallel to the plane of which the camera was placed) was provided by a cold light source (KL 2500 LED, Schott) which passed through a sheet of paper acting as a diffuser.

Optical profilometry and image analysis for quantifying the deposit morphology. The central deposit as well as the peripheral ring of the NP patterns obtained after drop drying were analyzed by optical profilometry (Wyko NT1100, Veeco). A 20x objective was used for scanning. Individually scanned areas were stitched together and a curvature and tilt correction were applied to correct for deviations from flat morphology. The $X, Y, Z$ coordinates of the corrected full image were stored in ASCII format before being converted into a matrix of height 
values $Z$ in a $(X, Y)$ space with a fixed pixel size of $0.907 \times 0.907 \mu \mathrm{m}^{2}$, using Matlab (The MathWorks).

The central deposit was detected using a threshold height value (typically $0.2 \mu \mathrm{m}$ ) and was 'cleaned' before taking the elliptical bounding box of the main region (Figure S2). Height values above $5 \mu \mathrm{m}$ were considered as aberrant and were removed. We then calculated the mean height of the elliptical region and the corresponding integrated volume. As we assumed a roughly circular shape for the deposit, the radius $R$ was taken as the mean of the ellipse radii. For the height profiles along the $X$ and $Y$ axes (Figure S3) we took the median of the profiles included in a centered region of width $R / 4$. For the ring patterns, the threshold step was sufficient for a correct detection of the structure. A similar height analysis was done here as well, except that we considered data points above $40 \mu \mathrm{m}$ as aberrant.

Measurement of surface tension of AzoTAB solutions under irradiation. The surface tension of solutions with varying AzoTAB concentrations (Figure S8) was measured with a DSA30 Drop Shape Analysis System (Krüss), by fitting the shape of pendant drop (volume 9.5 - $13.5 \mu \mathrm{L}$, depending on $C_{s}$ ) with the Young-Laplace equation. Each measurement was repeated three times. The experiments were conducted under light irradiation conditions similar to the patterning experiments. The pendant drop was formed under homogeneous white light irradiation (from a table top LED lamp). Approximately $91 \mathrm{~s}$ after drop formation, UV light (wavelength $365 \mathrm{~nm}$ ) was shined on the whole drop through a light guide coupled to the light source (CoolLED PreciseExcite). The distance between the tip and the light guide was $2 \mathrm{~cm}$, corresponding to a UV intensity $I_{U V} \sim 330 \mathrm{~W} / \mathrm{m}^{2}$. The surface tension was recorded as a function of time where $t=0$ corresponds to the time at which the drop was formed. All experiments were carried out at temperature $21.1 \pm 0.4{ }^{\circ} \mathrm{C}$ and relative humidity $33.5 \pm 0.5 \%$. 
For calculating the surface tension change due to UV irradiation $\Delta \gamma=\gamma_{W+U V}-\gamma_{W}$ (Figure 5), we averaged the values of the surface tension under white light illumination for a time period of $10 \mathrm{~s}$ before switching on the UV beam. For each AzoTAB concentration, we repeated this procedure three times and we finally calculated the mean value, $\gamma_{W}$. We next averaged the $\gamma$ values under both white and UV light exposure for a time period of $10 \mathrm{~s}$ after $\gamma$ reached its plateau value (Figure S8). To define the time at which the plateau was reached, we calculated the first derivative of $\gamma(t)$ and we took the point where $\mathrm{d} \gamma / \mathrm{d} t$ reached its first minimum. For each photosurfactant concentration, we repeated this procedure three times and we finally calculated the mean value, $\gamma_{W+U V}$.

\section{ASSOCIATED CONTENT}

Supporting Information. The Supporting Information is available free of charge on the ACS Publications website at DOI: XXX. Patterning in drops of different volumes (Figure S1), detection of the deposit with image analysis (Figure S2), typical height profiles (Figure S3), low magnification reflection microscopy images of deposits (Figures S4 - S7 and S9), time evolution of the surface tension of irradiated AzoTAB solutions (Figure S8), patterning on polymeric substrates (Figure S10) and flow visualization during drop evaporation under UV irradiation (Movie S1).

\section{AUTHOR INFORMATION}

\section{Corresponding Author}

*E-mail: damien.baig@ens.fr. Telephone: $\quad+33 \quad 1 \quad 4432 \quad 2405 . \quad$ Website: http://www.baigllab.com/. 


\section{Author Contributions}

$\dagger$ These authors contributed equally. MA, SNV and DB developed the concept of eOMA. MA and SNV performed the experiments and the data analysis. MM developed the MATLAB code for image analysis and contributed to the construction of the optical setup. SR helped in imaging (reflection images). The manuscript was written by MA, SNV and DB with contributions of all authors. All authors have given approval to the final version of the manuscript.

\$Current address: University of Luxembourg, Physics \& Materials Science Research Unit, 162a Avenue de la Faiencerie, Luxembourg L-1511, Luxembourg

\section{Funding Sources}

This work was supported by the Mairie de Paris [Emergence(s) 2012]. MA acknowledges funding from the European Commission (FP7-PEOPLE-2013-IEF / Project 624806 "DIOPTRA"). SNV acknowledges funding from the Department of Science and Technology, INDIA (DST) through INSPIRE faculty award (DST/INSPIRE/04/2015/000544). This work was performed in part in the technology platform of Institut Pierre-Gilles de Gennes (IPGG) with supports from “Investissements d'Avenir” for Labex and Equipex IPGG (ANR-10-LABX31 and ANR-10-IDEX-0001-02-PSL).

\section{Notes}

The authors declare no competing financial interest.

\section{ACKNOWLEDGMENT}

We thank Guillaume Schelcher and Guillaume Laffite for assistance with the optical profilometry experiments. 


\section{REFERENCES}

(1) Xia, Y.; Gates, B.; Yin, Y.; Lu, Y. Monodispersed Colloidal Spheres: Old Materials with New Applications. Adv. Mater. 2000, 12, 693-713.

(2) Yang, J.; Choi, M. K.; Kim, D.-H.; Hyeon, T. Designed Assembly and Integration of Colloidal Nanocrystals for Device Applications. Adv. Mater. 2016, 28, 1176-1207.

(3) Jiang, L.; Chen, X.; Lu, N.; Chi, L. Spatially Confined Assembly of Nanoparticles. Acc. Chem. Res. 2014, 47, 3009-3017.

(4) Kim, S.-H.; Lee, S. Y.; Yang, S.-M.; Yi, G.-R. Self-Assembled Colloidal Structures for Photonics. NPG Asia Mater. 2011, 3, 25-33.

(5) Karg, M.; König, T. A. F.; Retsch, M.; Stelling, C.; Reichstein, P. M.; Honold, T.; Thelakkat, M.; Fery, A. Colloidal Self-Assembly Concepts for Light Management in Photovoltaics. Mater. Today 2015, 18, 185-205.

(6) Shipway, A. N.; Katz, E.; Willner, I. Nanoparticle Arrays on Surfaces for Electronic, Optical, and Sensor Applications. ChemPhysChem 2000, 1, 18-52.

(7) Li, F.; Josephson, D. P.; Stein, A. Colloidal Assembly: The Road from Particles to Colloidal Molecules and Crystals. Angew. Chemie Int. Ed. 2011, 50, 360-388.

(8) Vogel, N.; Retsch, M.; Fustin, C.-A.; del Campo, A.; Jonas, U. Advances in Colloidal Assembly: The Design of Structure and Hierarchy in Two and Three Dimensions. Chem. Rev. 2015, 115, 6265-6311.

(9) Cui, Y.; Björk, M. T.; Liddle, J. A.; Sönnichsen, C.; Boussert, B.; Alivisatos, A. P. Integration of Colloidal Nanocrystals into Lithographically Patterned Devices. Nano 
Lett. 2004, 4, 1093-1098.

(10) Wilbur, J. L.; Kumar, A.; Kim, E.; Whitesides, G. M. Microfabrication by Microcontact Printing of Self-Assembled Monolayers. Adv. Mater. 1994, 6, 600-604.

(11) Kraus, T.; Malaquin, L.; Schmid, H.; Riess, W.; Spencer, N. D.; Wolf, H. Nanoparticle Printing with Single-Particle Resolution. Nat. Nanotechnol. 2007, 2, 570-576.

(12) Xu, L.; Robert, L.; Ouyang, Q.; Taddei, F.; Chen, Y.; Lindner, A. B.; Baigl, D. Microcontact Printing of Living Bacteria Arrays with Cellular Resolution. Nano Lett. 2007, 7, 2068-2072.

(13) Deegan, R. D.; Bakajin, O.; Dupont, T. F.; Huber, G.; Nagel, S. R.; Witten, T. A. Capillary Flow as the Cause of Ring Stains from Dried Liquid Drops. Nature 1997, $389,827-829$.

(14) Denkov, N. D.; Velev, O. D.; Kralchevsky, P. A.; Ivanov, I. B.; Yoshimura, H.; Nagayama, K. Two-Dimensional Crystallization. Nature 1993, 361, 26-26.

(15) Deegan, R. D.; Bakajin, O.; Dupont, T. F.; Huber, G.; Nagel, S. R.; Witten, T. A. Contact Line Deposits in an Evaporating Drop. Phys. Rev. E 2000, 62, 756-765.

(16) Majumder, M.; Rendall, C. S.; Eukel, J. A.; Wang, J. Y. L.; Behabtu, N.; Pint, C. L.; Liu, T.-Y.; Orbaek, A. W.; Mirri, F.; Nam, J.; Barron, A. R.; Hauge, R. H.; Schmidt, H. K.; Pasquali, M. Overcoming the "Coffee-Stain” Effect by Compositional Marangoni-Flow-Assisted Drop-Drying. J. Phys. Chem. B 2012, 116, 6536-6542.

(17) Talbot, E. L.; Yang, L.; Berson, A.; Bain, C. D. Control of the Particle Distribution in Inkjet Printing through an Evaporation-Driven Sol-Gel Transition. ACS Appl. Mater. Interfaces 2014, 6, 9572-9583. 
(18) Anyfantakis, M.; Baigl, D. Dynamic Photocontrol of the Coffee-Ring Effect with Optically Tunable Particle Stickiness. Angew. Chem. Int. Ed. Engl. 2014, 53, 1407714081.

(19) Anyfantakis, M.; Geng, Z.; Morel, M.; Rudiuk, S.; Baigl, D. Modulation of the CoffeeRing Effect in Particle/Surfactant Mixtures: The Importance of Particle-Interface Interactions. Langmuir 2015, 31, 4113-4120.

(20) Anyfantakis, M.; Baigl, D. Manipulating the Coffee-Ring Effect: Interactions at Work. ChemPhysChem 2015, 16, 2726-2734.

(21) Devineau, S.; Anyfantakis, M.; Marichal, L.; Kiger, L.; Morel, M.; Rudiuk, S.; Baigl, D. Protein Adsorption and Reorganization on Nanoparticles Probed by the CoffeeRing Effect: Application to Single Point Mutation Detection. J. Am. Chem. Soc. 2016, $138,11623-11632$.

(22) Kuncicky, D. M.; Velev, O. D. Surface-Guided Templating of Particle Assemblies inside Drying Sessile Droplets. Langmuir 2008, 24, 1371-1380.

(23) Anyfantakis, M.; Baigl, D.; Binks, B. P. Evaporation of Drops Containing Silica Nanoparticles of Varying Hydrophobicities: Exploiting Particle-Particle Interactions for Additive-Free Tunable Deposit Morphology. Langmuir 2017, 33, 5025-5036.

(24) Ray, M. A.; Kim, H.; Jia, L. Dynamic Self-Assembly of Polymer Colloids To Form Linear Patterns. Langmuir 2005, 21, 4786-4789.

(25) Lin, Z. Q.; Granick, S. Patterns Formed by Droplet Evaporation from a Restricted Geometry. J. Am. Chem. Soc. 2005, 127, 2816-2817.

(26) Fan, F.; Stebe, K. J. Assembly of Colloidal Particles by Evaporation on Surfaces with 
Patterned Hydrophobicity. Langmuir 2004, 20, 3062-3067.

(27) Harris, D. J.; Hu, H.; Conrad, J. C.; Lewis, J. A. Patterning Colloidal Films via Evaporative Lithography. Phys. Rev. Lett. 2007, 98, 148301.

(28) Harris, D. J.; Conrad, J. C.; Lewis, J. A. Evaporative Lithographic Patterning of Binary Colloidal Films. Philos. Trans. R. Soc. A Math. Phys. Eng. Sci. 2009, 367, 5157-5165.

(29) Baigl, D. Photo-Actuation of Liquids for Light-Driven Microfluidics: State of the Art and Perspectives. Lab Chip 2012, 12, 3637.

(30) Garnier, N.; Grigoriev, R. O.; Schatz, M. F. Optical Manipulation of Microscale Fluid Flow. Phys. Rev. Lett. 2003, 91, 54501.

(31) Georgiadis, A.; Routh, A. F.; Murray, M. W.; Keddie, J. L. Bespoke Periodic Topography in Hard Polymer Films by Infrared Radiation-Assisted Evaporative Lithography. Soft Matter 2011, 7, 11098-11102.

(32) Salas, J. A. V; van der Veen, J. M.; Michels, J. J.; Darhuber, A. A. Active Control of Evaporative Solution Deposition by Modulated Infrared Illumination. J. Phys. Chem. C 2012, 116, 12038-12047.

(33) Ta, V. D.; Carter, R. M.; Esenturk, E.; Connaughton, C.; Wasley, T. J.; Li, J.; Kay, R. W.; Stringer, J.; Smith, P. J.; Shephard, J. D. Dynamically Controlled Deposition of Colloidal Nanoparticle Suspension in Evaporating Drops Using Laser Radiation. Soft Matter 2016, 12, 4530-4536.

(34) Kumar, A.; Chuang, H.-S.; Wereley, S. T. Dynamic Manipulation by Light and Electric Fields: Micrometer Particles to Microliter Droplets. Langmuir 2010, 26, $7656-7660$. 
(35) Lin, L.; Peng, X.; Mao, Z.; Li, W.; Yogeesh, M. N.; Rajeeva, B. B.; Perillo, E. P.; Dunn, A. K.; Akinwande, D.; Zheng, Y. Bubble-Pen Lithography. Nano Lett. 2016, $16,701-708$.

(36) Sakaguchi, A.; Higashiguchi, K.; Yotsuji, H.; Matsuda, K. Photocontrol of Clustering, Retaining, and Releasing of Microbeads Concomitant with Phototransformation of Supramolecular Architecture of Amphiphilic Diarylethene. J. Phys. Chem. B 2017, $121,4265-4272$.

(37) Diguet, A.; Guillermic, R.-M.; Magome, N.; Saint-Jalmes, A.; Chen, Y.; Yoshikawa, K.; Baigl, D. Photomanipulation of a Droplet by the Chromocapillary Effect. Angew. Chem. Int. Ed. Engl. 2009, 48, 9281-9284.

(38) Chevallier, E.; Mamane, A.; Stone, H. a.; Tribet, C.; Lequeux, F.; Monteux, C. Pumping-out Photo-Surfactants from an Air-water Interface Using Light. Soft Matter 2011, 7, 7866-7874.

(39) Varanakkottu, S. N.; George, S. D.; Baier, T.; Hardt, S.; Ewald, M.; Biesalski, M. Particle Manipulation Based on Optically Controlled Free Surface Hydrodynamics. Angew. Chemie Int. Ed. 2013, 52, 7291-7295.

(40) Venancio-Marques, A.; Baigl, D. Digital Optofluidics: LED-Gated Transport and Fusion of Microliter-Sized Organic Droplets for Chemical Synthesis. Langmuir 2014, $30,4207-4212$.

(41) Kavokine, N.; Anyfantakis, M.; Morel, M.; Rudiuk, S.; Bickel, T.; Baigl, D. LightDriven Transport of a Liquid Marble with and against Surface Flows. Angew. Chemie Int. Ed. 2016, 55, 11183-11187. 
(43) Georgiadis, A.; Routh, A. F.; Murray, M. W.; Keddie, J. L. Bespoke Periodic Topography in Hard Polymer Films by Infrared Radiation-Assisted Evaporative Lithography. Soft Matter 2011, 7, 11098-11102.

(44) Feldmann, D.; Maduar, S. R.; Santer, M.; Lomadze, N.; Vinogradova, O. I.; Santer, S. Manipulation of Small Particles at Solid Liquid Interface: Light Driven Diffusioosmosis. Sci. Rep. 2016, 6, 36443.

(45) Still, T.; Yunker, P. J.; Yodh, A. G. Surfactant-Induced Marangoni Eddies Alter the Coffee-Rings of Evaporating Colloidal Drops. Langmuir 2012, 28, 4984-4988.

(46) Diguet, A.; Mani, N. K.; Geoffroy, M.; Sollogoub, M.; Baigl, D. Photosensitive Surfactants with Various Hydrophobic Tail Lengths for the Photocontrol of Genomic DNA Conformation with Improved Efficiency. Chem. Eur. J. 2010, 16, 11890-11896. 


\section{Graphical Table of Contents (ToC):}

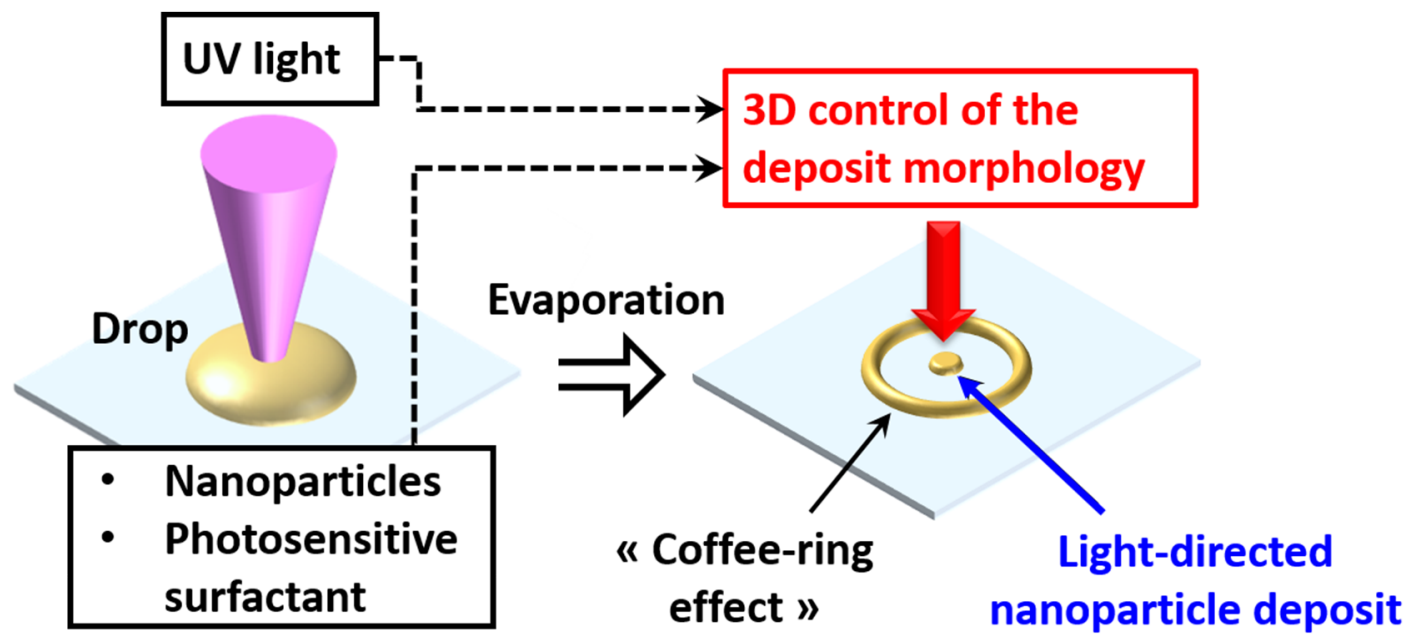

